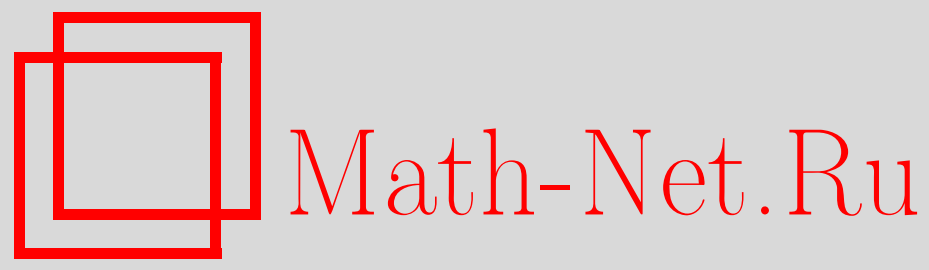

С. Н. Набоко, Теорема Зигмунда и граничное поведение операторных $R$-функций, Функи. анализ и его прил., 1996, том 30, выпуск 3, 82-84

DOI: https://doi.org/10.4213/faa540

Использование Общероссийского математического портала MathNet.Ru подразумевает, что вы прочитали и согласны с пользовательским соглашением

http://www . mathnet.ru/rus/agreement

Параметры загрузки:

IP : 34.239 .49 .27

26 апреля 2023 г., 16:11:07

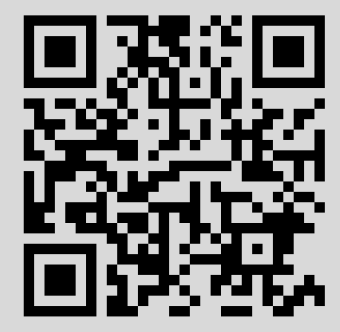




\title{
Теорема Зигмунда и граничное поведение операторных $R$-функций
}

\author{
(c) 1996. С. Н. НАьоко ${ }^{1}$
}

В работе изучается граничное поведение аналитических в полуплоскости $\mathbb{C}_{+}$ оператор-функций $T(\lambda)$, принимающих значения в классе диссипативных операторов $\left(\operatorname{Im} T(\lambda) \stackrel{\text { def }}{=}\left(T^{*}(\lambda)-T(\lambda)\right) / 2 i \geqslant 0, \lambda \in \mathbb{C}_{+}\right)$в гильбертовом пространстве $H$. Функции такого типа называются операторными $R$-функциями и появляются в различных задачах теории операторов в гильбертовом пространстве и в математической физике: в теории возмущений сингулярного спектра самосопряженных операторов [1, 2], теории рассеяния [3], в задачах, связанных с формулой следа $[4,5]$, в теории функциональных моделей и характеристических функций несамосопряженных операторов $[4,6]$, теории вольтерровых операторов [7], при изучении преобразования Гильберта в пространстве векторнозначных функций [8] и в теории мартингалов.

Основные факты, касающиеся граничного поведения на $\mathbb{R}$ операторных $R$-функций, принимающих значения в классах $\mathfrak{S}_{p}, 0<p<\infty$, компактных операторов, $s$-числа которых суммируемы со степенью $p[9]$, приведены в работе [10]. Как показано в [2], операторная $R$-функция $T(\lambda)$ общего вида может быть представлена в следующей форме:

$$
T(\lambda)=A+B \lambda+\left.V^{1 / 2}(I+\lambda \mathscr{L})(\mathscr{L}-\lambda)^{-1} V^{1 / 2}\right|_{H},
$$

где $A=A^{*}, B \geqslant 0$ - ограниченные операторы в $H$, а $V \geqslant 0$ и $\mathscr{L}=\mathscr{L}^{*}$ - операторы в более широком вспомогательном гильбертовом пространстве $\mathscr{H} \supset H$, причем $\left.V\right|_{\mathscr{H} \ominus H}=0$. Здесь оператор $\mathscr{L}$ является обобщением дилатации максимального диссипативного оператора [6] на случай общей операторной $R$-функции. Легко видеть, что если $T(i) \in \mathfrak{S}_{p}$, то $A, B, V \in \mathfrak{S}_{p}, p>0$, и, следовательно, $T(\lambda) \in \mathfrak{S}_{p}, \lambda \in \mathbb{C}_{+}$. Однако вопрос о существовании п.в. (нетангенциальных) граничных значений $T(k+i 0)$ и топология предельного перехода на границу представляют значительную трудность. Формула (1) позволяет без уменьшения общности ограничиться лишь операторными $R$-функциями более специального вида

$$
T(\lambda)=\left.V^{1 / 2}(\mathscr{L}-\lambda)^{-1} V^{1 / 2}\right|_{H} .
$$

При этом различие между пространствами $H$ и $\mathscr{H}$ представляется здесь несущественным; поэтому достаточно для простоты записи считать все операторы действующими в $H$. Известно [10], что при $V \in \mathfrak{S}_{p}, p<1$, п.в. на $\mathbb{R}$ существуют нетангенциальные граничные пределы $T(k+i 0), k \in \mathbb{R}$, в топологии пространства $\mathfrak{S}_{p}$. В то же время для любого неотрицательного оператора $V \notin \mathfrak{S}_{1}$ можно найти $\mathscr{L}=\mathscr{L}^{*}$, такой, что $T(\lambda)$ (см. (2)) не имеет всюду

\footnotetext{
${ }^{1}$ Работа была выполнена при поддержке INTAS, грант 93.1815.
} 
на $\mathbb{R}$ «радиальных» граничных пределов даже в слабой топологии [11]. Однако если $V \in \mathfrak{S}_{1}$, то граничные пределы существуют в метрике пространства $\mathfrak{S}_{p}$ для любого $p>1$, но, вообще говоря, не в топологии сходимости по ядерной норме [10]. При изучении данного круга вопросов большую роль играет аналогия с действием преобразования Гильберта в классе скалярных функций. Более того, она служит одним из источников доказательств операторных теорем [10]. Так, известная теорема Колмогорова [12] о действии преобразования Гильберта в $L^{p}, 0<p<1$, имеет прямым аналогом ранее упомянутый результат о граничных значениях операторных $R$-функций при $V \in \mathfrak{S}_{p}$. Слабый тип $(1,1)$ преобразования Гильберта в $L^{1}$ влечет за собой соответствующее утверждение для $V \in \mathfrak{S}_{1}$. Переходя к важному в приложениях вопросу о существовании нетангенциальных граничных пределов для $T(\lambda)$ в ядерной норме, можно упомянуть теорему Зигмунда о классе $L \log L$ : скалярная $R$-функция $f(z)$ в круге $|z|<1$ принадлежит классу Харди $H^{1}(\mathbb{D}) \Longleftrightarrow \operatorname{Im} f\left(e^{i \theta}\right) \in L \log L$ (т.е. $\left.\int_{0}^{2 \pi}\left|\operatorname{Im} f\left(e^{i \theta}\right)\right| \log ^{+}\left(1 /\left|\operatorname{Im} f\left(e^{i \theta}\right)\right|\right) d \theta<\infty\right)[12]$. Последнее позволяет ввести естественное условие на собственные числа оператора $V$ :

$$
\sum_{n} s_{n}(V) \log ^{+}\left(1 / s_{n}(V)\right)<\infty
$$

и поставить вопрос о ядерности граничных пределов $T(k+i 0)$ при выполнении условия (3) на «возмущение» $V$, более сильного, чем $V \in \mathfrak{S}_{1}$. Кроме того, параллельно рассмотрим еще два других ограничения на $V$ :

$$
\begin{gathered}
V \in l_{1} \times l_{\omega} \stackrel{\text { def }}{\Longleftrightarrow} \exists\left\{\alpha_{n}\right\}_{n=1}^{\infty} \in l_{1}, \alpha_{n} \downarrow 0,\left\{\beta_{n}\right\}_{n=1}^{\infty}, \beta_{n} \downarrow 0, \sum_{n} \frac{\beta_{n}}{n}<\infty, \\
s_{n}(V) \leqslant \alpha_{n} \beta_{n}, \\
\sum_{n} \frac{s_{n}^{1 / 2}(V)}{\sqrt{n}}<\infty .
\end{gathered}
$$

В работе [10] доказано, что из условия (4) следует существование п.в. на $\mathbb{R}$ нетангенциальных граничных пределов $T(k+i 0)$ в норме пространства $\mathfrak{S}_{1}$. Легко проверить, что справедливы следующие импликации: $(4) \Longrightarrow(3),(4) \Longrightarrow(5)$, причем обе импликации, как показывают соответствующие контрпримеры, вообще говоря, необратимы. Кроме того, условие (3) эквивалентно сходимости ряда $\sum_{n} s_{n}(V) \ln n$, и ни одно из условий $(3),(5)$ не влечет за собой другое. Наконец, из всех трех условий вытекает, что $s_{n}(V) n \rightarrow 0, n \rightarrow \infty$. Если же дополнительно известно, что $s_{n}(V) n$ стремится к 0 монотонно, то все три условия будут эквивалентны друг другу.

Оказывается, что естественный операторный аналог теоремы Зигмунда для $R$-функций несправедлив.

Теорема 1. Существует неотрицательный компактный оператор $V$, удовлетворяющий условию (3), такой, что для подходящего самосопряженного оператора $\mathscr{L}$ в $H$ граничнье значения $T(k+i 0)$ не принадлежат классу $\mathfrak{S}_{1}$ для п.в. $k \in \mathbb{R}$. 
Заметим, что из условия (3) следует, что $T(k+i 0) \in \bigcap_{p>1} \mathfrak{S}_{p}$ для п.в. $k \in \mathbb{R}$. Таким образом, достаточное для ядерности оператора $T(k+i 0)$ (для п.в. $k \in \mathbb{R}$ ) условие (4) не может быть ослаблено до «условия Зигмунда» (3). Отметим, что условие (5) также не влечет за собой принадлежность $T(k+i 0) \in \mathfrak{S}_{1}$ для п.в. $k \in \mathbb{R}[10]$.

Далее рассмотрим вопрос о граничных значениях ядернозначных операторных $R$-функций $\left(V \in \mathfrak{S}_{1}\right)$. Отметим, что в «классах Лоренца» $\mathfrak{S}_{\pi}[9]\left(\pi=\left\{\pi_{n}\right\}_{n=1}^{\infty}\right.$, $\left.\pi_{n} \downarrow 0\right)$ в работе [10] был получен окончательный ответ. Для произвольной ядернозначной операторной $R$-функции $T(\lambda)$ ее граничные значения $T(k+i 0)$ принадлежат $\mathfrak{S}_{\pi}$ для п.в. $k \in \mathbb{R} \Longleftrightarrow \sum_{n} \pi_{n} / n<\infty$. Ниже будет рассмотрен вопрос о граничных значениях в «классах Марцинкевича» $\mathfrak{S}_{\Pi}[9]$, сопряженных к «классам Лоренца» $\left(T \in \mathfrak{S}_{\Pi} \Longleftrightarrow \sup _{n} \sum_{k=1}^{n} s_{k}(T) / \sum_{k=1}^{n} \pi_{k}<\infty\right)$.

Teоpema 2. (1) Пусть $C_{n}>0, C_{n} \uparrow \infty, n \rightarrow \infty u \sum_{n} 1 /\left(n C_{n}\right)<\infty ;$ тогда у ядернозначной операторной $R$-функции $T(\lambda)$ п.в. на $\mathbb{R}$ существуют нетангенциальные граничнье предель в норме $\sup _{n} \sum_{k=1}^{n} s_{k}(T) / C_{n}$ (cм. [10]).

(2) Пусть $C_{n}>0, C_{n} \uparrow \infty, n \rightarrow \infty, u$ для любой операторной $R$-функиии $T(\lambda)$ вида (2) с $V \in \mathfrak{S}_{1}$ граничные значения для п.в. $k \in \mathbb{R}$ удовлетворяют условию $\sup _{n} \sum_{k=1}^{n} s_{k}(T(k+i 0)) / C_{n}<\infty ;$ тогда $C_{n} \geqslant C_{0} \ln n, n=2,3, \ldots$, где постолнная $C_{0}$ больше 0 .

Отметим, что из условий на последовательность $C_{n}$ из п. (1) теоремы 2 не вытекает оценки, лучшей, чем $\ln n / C_{n}=o(1), n \rightarrow \infty$.

Таким образом, граничные значения ядернозначных операторных $R$-функций не могут, вообще говоря, находиться в «классе Марцинкевича» $\mathfrak{S}_{\Pi}$, «лучшем», чем $\mathfrak{S}_{\Omega} \equiv \mathfrak{S}_{\omega}^{*}-$ сопряженный к классу Мацаева $\mathfrak{S}_{\omega}[9]\left(\mathfrak{S}_{\Omega} \equiv \mathfrak{S}_{\Pi}\right.$ при $\left.\pi_{n}=1 / n\right)$.

\section{ЛитературА}

1. Faddeev L. D., Pavlov B. S. Lecture Notes in Math., Vol. 1043, Springer-Verlag (1984), p. 124-128. 2. Naboko S. N. Arkiv Mat., 25, 115-140 (1987). 3. Набоко C. H. Проблемы матем. физ., ЛГУ, 12, 132-155 (1987). 4. Веселов В. Ф., Набоко С. Н. Матем. сб., 129 (171), №1, 20-29 (1986). 5. Бирман М. С., Яфаев Д. Р. Алгебра и анализ, 4, №5, 1-44 (1992). 6. Сёкефальви-Надь Б., Фойаш Ч. Гармонический анализ операторов в гильбертовом пространстве. Мир, М., 1970. 7. Гохберг И. Ц., Крейн М. Г. Теория вольтерровых операторов в гильбертовом пространстве. Наука, M., 1967. 8. Bourgain J. Israel Semin. Geom. Funct. Anal., S.I., XVI1-XVI23 (1984). 9. Гохберг И. Ц., Крейн М. Г. Введение в теорию линейных несамосопряженных операторов. Наука, М., 1965. 10. Набоко C. H. Алгебра и анализ, 1, №5, 197-222 (1989). 11. Naboko S. N. Func. Anal. and Oper. Theory, Banach Center Publ., 30, 277-285 (1994). 12. Кусис П. Введение в теорию пространств $H^{p}$. Мир, М., 1984 . 